\title{
Diffusion tensor imaging of the C1-C3 dorsal root ganglia and greater occipital nerve for cervicogenic headache
}

\author{
Lang Wang ${ }^{1, *}$, Jiang Shen ${ }^{1, *}$, Sushant Das $^{2}$, and Hanfeng Yang ${ }^{3}$ \\ 'Department of Radiology, West China School of Public Health and West China Fourth Hospital, Sichuan University, Chengdu, China \\ ${ }^{2}$ North Sichuan Medical College, Nanchong, China \\ ${ }^{3}$ Department of Pain, Affiliated Hospital of North Sichuan Medical College, Nanchong, China
}

Received March 31, 2020

Revised May 16, 2020

Accepted May 22, 2020

Handling Editor: Francis Sahngun Nahm

\section{Correspondence}

Hanfeng Yang

Department of Pain, Affiliated Hospital of North Sichuan Medical College, 63 Wenhua Road, Nanchong, Sichuan

Province 637000, China

Tel: +86-13540652806

Fax: +86-0817-2222856

E-mail:yhf_nsmc@163.com

*These authors contributed equally to this work.
Background: Previous studies showed neurography and tractography of the greater occipital nerve (GON). The purpose of this study was determining diffusion tensor imaging (DTI) parameters of bilateral GONs and dorsal root ganglia (DRG) in unilateral cervicogenic headache as well as the grading value of DTI for severe headache. The correlation between DTI parameters and clinical characteristics was evaluated. Methods: The fractional anisotropy (FA) and apparent diffusion coefficient (ADC) values in bilateral GONs and cervical DRG (C2 and C3) were measured. Grading values for headache severity was calculated using a receiver operating characteristics curve. The correlation was analyzed with Pearson's coefficient.

Results: The FA values of the symptomatic side of GON and cervical DRG (C2 and C3) were significantly lower than that of the asymptomatic side (all the $P<0.001$ ), while the ADC values were significantly higher $(P=0.003, P<0.001$, and $P=0.003$, respectively). The FA value of 0.205 in C2 DRG was considered the grading parameter for headache severity with sensitivity of 0.743 and specificity of $0.999(P<$ 0.001). A negative correlation and a positive correlation between the FA and ADC value of the GON and headache index $(\mathrm{HI} ; r=-0.420, P=0.037$ and $r=0.531, P=$ 0.006 , respectively) was found.

Conclusions: DTI parameters in the symptomatic side of the C2 and C3 DRG and GON were significantly changed. The FA value of the C2 DRG can grade headache severity. DTI parameters of the GON significantly correlated with HI.

Key Words: Cervical Vertebrae; Chronic Pain; Diffusion Tensor Imaging; Ganglia, Spinal; Headache; Magnetic Resonance Imaging; ROC Curve; Sensitivity and Specificity.

\section{INTRODUCTION}

A cervicogenic headache (CGH) is classified as a secondary headache arising from degenerative cervical spine disorders, which is described as a prolonged unilateral headache with a prevalence rate of at least $70 \%$ is reported during an individual's lifetime history of headache [1].
CGH is considered to be a kind of referred pain from cervical soft tissue innervated by cervical segments spinal nerves, and is highly correlated with neuropathy in the C2C3 dorsal root ganglia (DRG), and entrapment of the upper three cervical spinal nerves branches, such as the greater occipital nerve (GON) [2].

The GON originates from the dorsal ramus of the $\mathrm{C} 2 \mathrm{spi}$ - (c) This is an open-access article distributed under the terms of the Creative Commons Attribution Non-Commercial License (http://creativecommons.org/licenses/by-nc/4.0/), which permits unrestricted non-commercial use, distribution, and reproduction in any medium, provided the original work is properly cited.

(c) The Korean Pain Society, 2020
Author contributions: Lang Wang: Writing/manuscript preparation; Jiang Shen: Writing/manuscript preparation; Sushant Das: Writing/manuscript preparation; Hanfeng Yang: Methodology. 
nal nerve, and then passes between the atlas and the axis, around the obliquus capitis inferior muscle, and semispinalis capitis [3]. Some segments of the course of the GON are thought to make the GON vulnerable to injury and entrapment [4]. The DRG carry sensory information from peripheral nerves. Intervertebral disc degeneration, as well as nerve root inflammation and compression can result in neuropathy of the upper three cervical DRG, which are recognized as an important cause of headaches in the occipital region $[5,6]$. Blocks of the GON and pulsed radiofrequency of the DRG are the most frequently used methods in the treatment of CGH $[4,6,7]$. However, the mechanisms of such invasive procedures are still unclear and lack objective evaluation criteria $[7,8]$. The diagnostic criteria proposed by the International Headache Society (IHS) and the Cervicogenic Headache International Study Group (CHISG) are widely used for CGH with different decisions $[2,3,9]$. Due to the non-specific signs of CGH, including cervical spine dysfunction and degeneration, it is difficult to obtain radiographic evidence in patients with $\mathrm{CGH}$.

As an advanced noninvasive form of magnetic resonance imaging (MRI), diffusion tensor imaging (DTI) can depict the nerve fibre tract, revealing exquisite details of the tissue microstructure [10], which can be a specific method for neuropathy diagnosis. Due to the different diffusion directions of free water molecules in three-dimensional space, anisotropy can be detected in the oriented structural properties of nerve fibre tracts. Fractional anisotropy (FA) and apparent diffusion coefficient (ADC) are generally applied in DTI for peripheral nerve entrapment evaluation [10-12]. Neurography and evaluation of GON tracts by tractography has been reported $[13,14]$. However, none of them tend to consider neuropathy of the DRG or grading the severity in patients with CGH by DTI. Due to the difficulty of accurately setting a region of interest (ROI) on these tiny nerves, as well as the long scanning time, DTI studies on CGH are still rare.

The aims of this study were to determine the DTI parameters changes of bilateral GONs and DRG in unilateral $\mathrm{CGH}$, as well as the grading value of DTI for severe headache. We also determined the correlation of DTI parameters with the headache index (HI), and the course of the disease (the period elapsed since the symptom started).

\section{MATERIALS AND METHODS}

\section{Patients}

Twenty-six patients with suspected unilateral CGH according to the CHISG criteria [15] in Affiliated Hospital of North Sichuan Medical College were included in this ret- rospective study from October 2017 to June 2019. They underwent a head-neck MRI examination, before receiving a diagnostic block of symptomatic side of the GON using landmark technique. The patients whose symptoms were relieved after the diagnostic block (a positive response) met the inclusion criteria. The clinical data included sex, age, pain laterality, headache frequency, course (from the CGH beginning to MRI examination), duration (hr/day), and headache intensity (using the numeric rating scale, NRS). Then, the HI (frequency $\times$ duration $\times$ NRS score) was calculated. Severe pain was identified when an NRS score $\geq 6$ from headache intensity was reached during the course of GCH $[16,17]$. The exclusion criteria were as follows: inability to undergo magnetic resonance scanning (including unsuitability for images analysis), inability to localize the pain laterality, inability to quantify the headache intensity, head-neck injury, hypertension, tumor, tension-type headache, or cluster headache. The asymptomatic sides of the nerves, in the patients, were used as the control group. This study was approved by the Institutional Review Board (IRB) of Affiliated Hospital of North Sichuan Medical College prior to initiation (IRB Number: 2017-183).

\section{MRI scanning and imaging analysis}

\section{1) MRI scanning}

Occipital MRI scanning was performed from the suboccipital area to the C4 level. MRI was performed on a GE Discovery ${ }^{\mathrm{TM}}$ MR750 3.0T system (GE Healthcare, Chicago, IL) using a 32-channel head coil. three-dimensional fast spin-echo $\mathrm{T}_{2}$-weighted imaging cube $\left(\mathrm{T}_{2} \mathrm{WI}\right.$ CUBE; GE Healthcare) (coronal scanning, thickness $=1 \mathrm{~mm}$; matrix $=192 \times 192$; field of vision $[\mathrm{FOV}]=216 \times 216$; flip angle $=90^{\circ}$; repetition time $[\mathrm{TR}]=2,500 \mathrm{msec}$; echo time $[\mathrm{TE}]$ = $69 \mathrm{msec}$ ) was used to observe the GON after a multislice reconstruction. A single-shot echo-planar imaging (thickness $=1.8 \mathrm{~mm}$; matrix $=64 \times 100 ; \mathrm{FOV}=200 \times 200$; flip angle $=90^{\circ} ; \mathrm{TR}=8,500 \mathrm{msec}$; TE $=57 \mathrm{msec}$; number of excitations $[\mathrm{NEX}]=6$; b value $=1,000 \mathrm{sec} / \mathrm{mm}^{2}$; direction 6 ; voxel size $=2 \mathrm{~mm}$, isotropic) was practiced for DTI. Axial $\mathrm{T}_{1}$-weighted imaging $\left(\mathrm{T}_{1} \mathrm{WI}\right.$, thickness $=1.8 \mathrm{~mm}$; matrix $=$ $256 \times 256 ; \mathrm{FOV}=200 \times 200 ;$ flip angle $=9^{\circ} ; \mathrm{TR}=6 \mathrm{msec} ; \mathrm{TE}$ $=2 \mathrm{msec}$ ) was performed to set the ROI. The ROI was also used for tractography. The threshold of the noise level was 40-60 and upper limit was 3,500-3,800, processed in the console. Other DTI metrics of the fibre track, such as max steps (160), min FA (0.18), max ADC (0.01) and reformat spacing (1.81931) were used as the default. 


\section{2) Imaging analysis}

After receiving training on GON and DRG anatomy, two observers, who were unaware of the patients' diagnosis, independently designated the ROI on bilateral GONs and the C2-C3 DRG at the GE workstation (Adw 4.4; GE Healthcare) to measure the FA and ADC values. The observers were two radiologists (doctors with 7 and 3 years of experience in MRI, respectively). The details of the method used and the processing steps for ROI delineation and DTI parameters are as follows: (1) the target segment of the proximal GON was determined by the border of the oblique

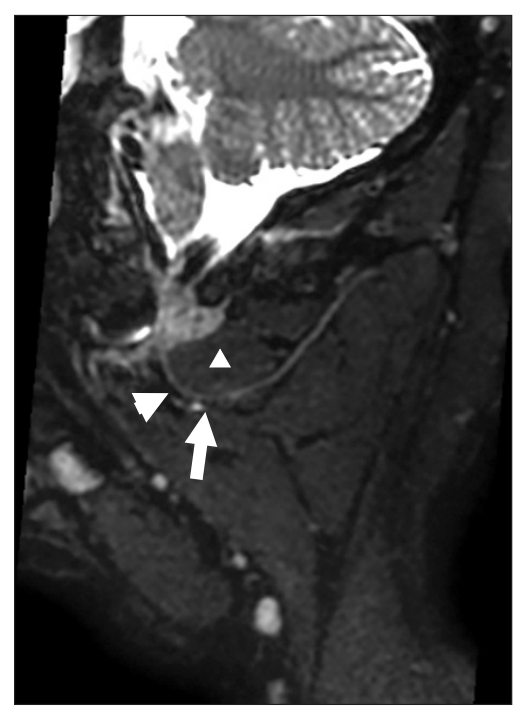

Fig. 1. Multi-slice reconstruction on $\mathrm{T}_{2}$-weighted imaging CUBE showed the greater occipital nerve (GON) and muscles around. We firstly found the oblique capitis inferior muscle (white triangle) and its proximal inferior border (arrow). Then we recognized the proximal GON (arrowhead) as the target nerve for subsequent post-processing step. capitis inferior muscles on the $\mathrm{T}_{2} \mathrm{WI}$ CUBE multi-slice reconstruction, which was considered as a possible entrapment section [18] (Fig. 1). And the C2-C3 DRG were found near the foramen on the axial $\mathrm{T}_{2} \mathrm{WI}$ CUBE; (2) the GON (on the $\mathrm{T}_{2} \mathrm{WI}$ CUBE reconstruction) and DRG (on the axial $\mathrm{T}_{2} \mathrm{WI}$ CUBE) were then identified on the axial $\mathrm{T}_{1} \mathrm{WI}$, and were coordinately localized at the post-processing step (Fig. 2); (3) visualization of the nerves on the axial $\mathrm{T}_{1} \mathrm{WI}$ at two close slices was subsequently confirmed; (4) the ROIs are manually drawn using the freehand tool to eliminate irrelevant fat and muscle tissues; (5) tractography was then performed based on the aforementioned ROIs setting, and the fibre tract is acquired to ensure that they have focused on the target GON (Figs. 3, 4); (6) eventually, the ROIs were delineated on the anatomical imaging and the FA and $A D C$ values were measured on the superimposed morphological axial $\mathrm{T}_{1} \mathrm{WI}$. For each FA or ADC, the two ROIs were set depending on the size of the nerve (ranging from 4.0$7.0 \mathrm{~mm}^{2}$, with a pixel value of 4-9) and the average value of each segment was calculated. For the control group, DTI parameters were acquired from the asymptomatic side of each patient. The time interval between obtaining two DTI parameters, by observer 1, was about a week.

\section{Statistical analysis}

The PASW Statistics ver. 18 (IBM Corp., Armonk, NY) was used for data analysis. The mean \pm standard deviation was used to present continuous variables. The $t$-test was used for comparison of bilateral DTI parameters. Statistical significance was considered when a two-tailed $P$ value was lower than 0.05 . A homogeneity of variance test was performed using Levene's test. Intra-class correlation coefficient (ICC) was used to evaluate inter-/intra-observer
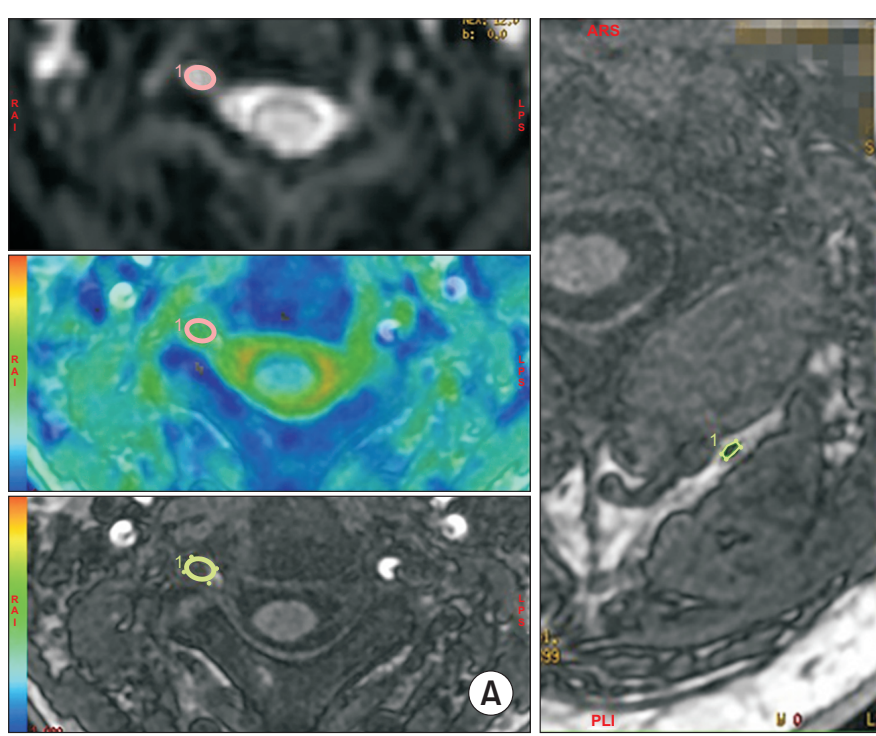

Fig. 2. Axial image showed the superimposed morphological $\mathrm{T}_{1}$-weighted imaging of right C3 dorsal root ganglia (DRG) (A) and left greater occipital nerve (GON) (B) with region of interest placement. The DRG was identified by directly visualization on original image $(b=0)$ and the target GON was identified with the help of aforementioned multi-slice reconstruction. 


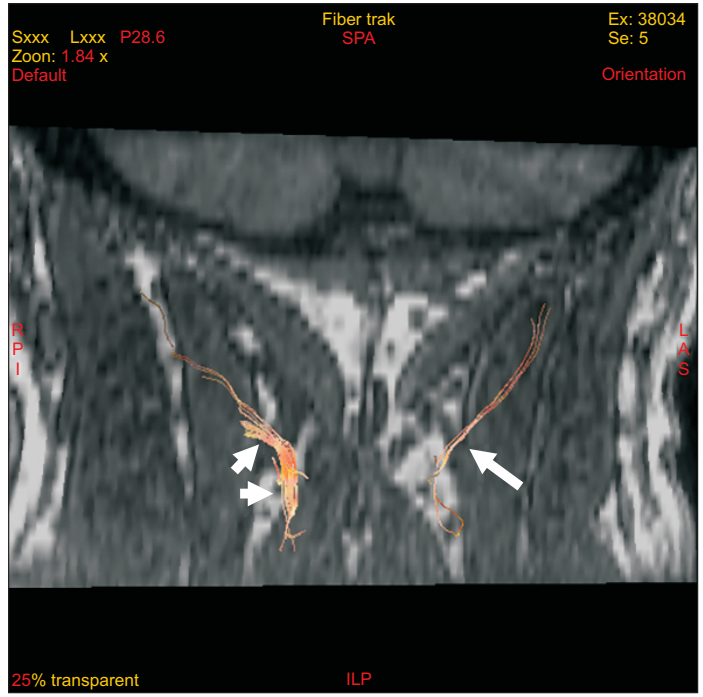

Fig. 3. Fibre tractography showed the right greater occipital nerve (arrowheads) was thicker and disordered than the left (arrows) in a 48-yearold male with right cervicogenic headache for 1 month.

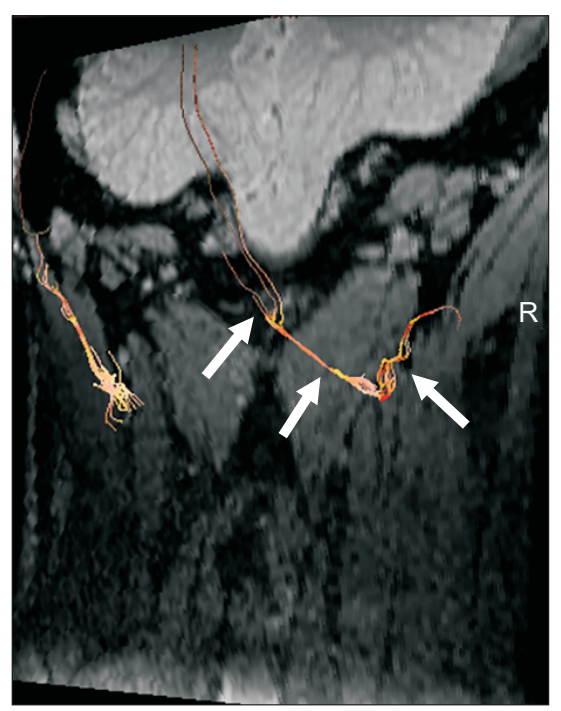

Fig. 4. A tractography of a 24-year-old male healthy volunteer showed the fibre tract of bilateral side greater occipital nerves (GONs). The fibre tract of normal GON showed continuous, smooth and clearly visualization of all the segments (arrows).

performance (excellent agreement, $>0.81$; substantial agreement, 0.60-0.79) [19]. We used a receiver operating characteristics (ROC) curve analysis to evaluate grading values by calculating the area under the curve (AUC) with a $95 \%$ confidence interval $(95 \% \mathrm{CI})$. The cut-off value was obtained by the maximum value of the Yoden index. The sensitivity, specificity, positive predictive value (PPV), and negative predictive value (NPV) for the cut-off value were also calculated. And the correlation of the course, and HI with the FA and ADC values was assessed using Pearson's coefficient.

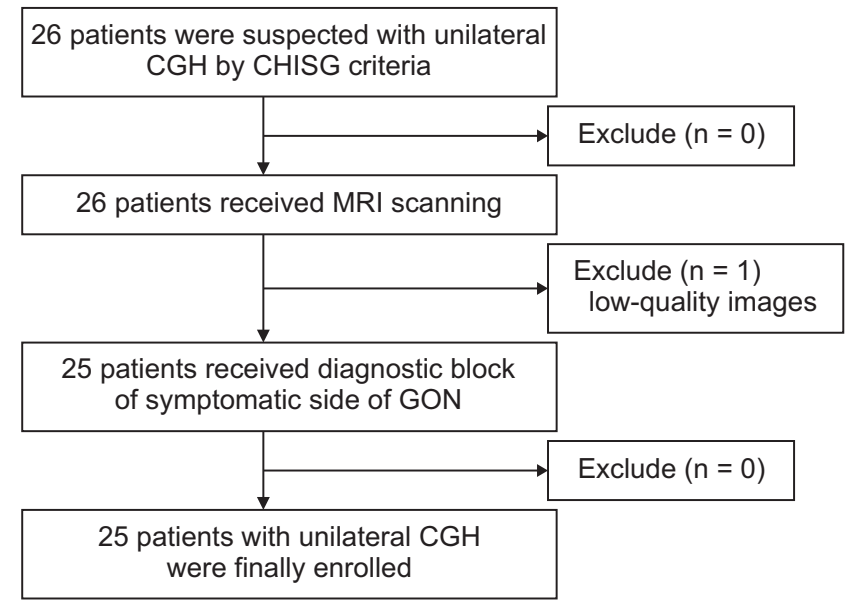

Fig. 5. A diagram about the enrollment of the patients. CGH: cervicogenic headache, CHISG: Cervicogenic Headache International Study Group, MRI: magnetic resonance imaging, GON: greater occipital nerve.

Table 1. The Clinical Characteristics of the Included Patients

\begin{tabular}{lc}
\hline \multicolumn{1}{c}{ Characteristic } & Value \\
\hline Sex (female/male) & $18 / 7$ \\
Mean age (yr) & $51.5 \pm 14.0(29-82)$ \\
Headache index & $897.8 \pm 750.3$ \\
Frequency of headache in a month (day) & $6.8 \pm 5.2$ \\
Course of disease (wk with headache) & $137.8 \pm 150.0$ \\
Duration (hr/day) & $6.3 \pm 3.6$ \\
Intensity (NRS score) & $6.1 \pm 1.5$ \\
Laterality of pain (left/right) & $9 / 16$ \\
\hline
\end{tabular}

The values are presented as number only, mean \pm standard deviation (range), or mean \pm standard deviation.

NRS: numeric rating score.

\section{RESULTS}

\section{Patients selection}

All 26 patients received magnetic resonance scanning but one of them was excluded due to low quality images (artifact). The other 25 patients received a diagnostic block of the symptomatic side of the GON, and all of them had a positive response. Finally, 25 patients with unilateral CGH were enrolled. A diagram about the enrollment of the patients is shown in Fig. 5. The patients' clinical features of are summarized in Table 1.

\section{FA and ADC values of the GON and DRG}

All the GON tractography was acquired with high quality. The homogeneity of variance test was found $(P>0.05)$ using Levene's test. The mean FA value of the GON was significantly lower for the symptomatic side, at $0.210 \pm$ 0.061 , than for the asymptomatic side at $0.326 \pm 0.053(P<$ $0.001)$. The mean ADC value $\left(\mathrm{mm}^{2} / \mathrm{sec} \times 10^{-3}\right)$ of the GON 
Table 2. DTI Parameters and the Observer Performance of Bilateral GON and C2-C3 DRG

\begin{tabular}{|c|c|c|c|c|c|}
\hline Variable & Symptomatic side & Asymptomatic side & $P$ value $(\mathrm{t})$ & Intra-observer ICC & Inter-observer ICC \\
\hline \multicolumn{6}{|l|}{$\mathrm{FA}$} \\
\hline C2 DRG & $0.176 \pm 0.059$ & $0.267 \pm 0.049$ & $<0.001(5.862)$ & 0.919 & 0.885 \\
\hline C3 DRG & $0.182 \pm 0.051$ & $0.261 \pm 0.048$ & $<0.001(5.605)$ & 0.914 & 0.861 \\
\hline GON & $0.210 \pm 0.061$ & $0.326 \pm 0.053$ & $<0.001(7.206)$ & 0.964 & 0.931 \\
\hline \multicolumn{6}{|c|}{$\operatorname{ADC}\left(\mathrm{mm}^{2} / \mathrm{sec} \times 10^{-3}\right)$} \\
\hline C2 DRG & $1.095 \pm 0.220$ & $0.772 \pm 0.300$ & $<0.001(4.336)$ & 0.924 & 0.909 \\
\hline C3 DRG & $1.038 \pm 0.295$ & $0.775 \pm 0.294$ & $0.003(3.160)$ & 0.934 & 0.966 \\
\hline GON & $0.649 \pm 0.204$ & $0.470 \pm 0.195$ & $0.003(3.167)$ & 0.967 & 0.953 \\
\hline
\end{tabular}

Values are presented as mean \pm standard deviation.

DTI: diffusion tensor imaging, GON: greater occipital nerve, DRG: dorsal root ganglia, ICC: intra-class correlation coefficient, FA: fractional anisotropy, ADC: apparent diffusion coefficient.

A

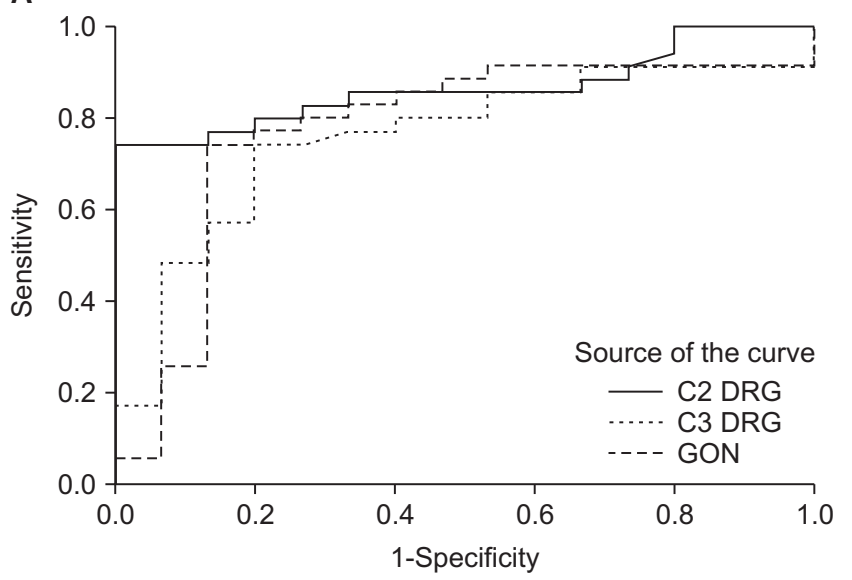

B

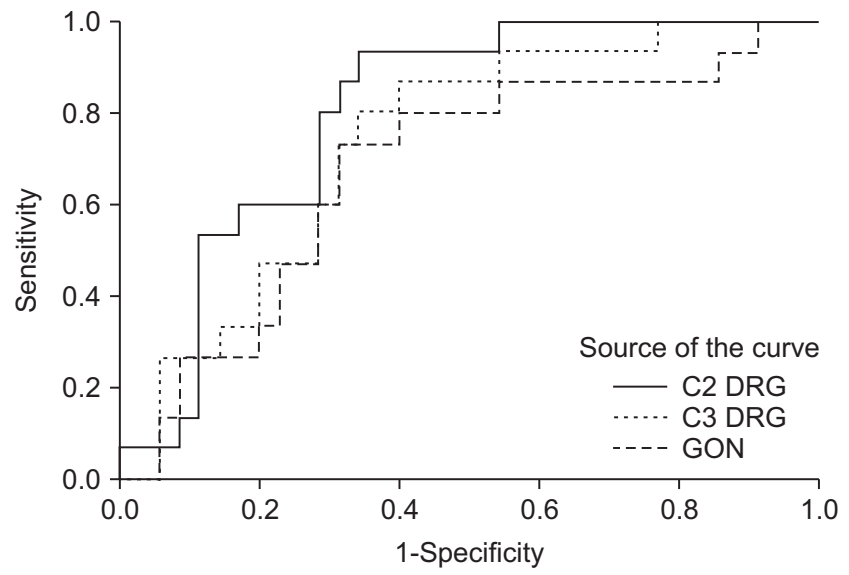

Fig. 6. Graphs showed receiver operating characteristics (ROC) curves analysis of (A) fractional anisotropy and (B) apparent diffusion coefficient values, respectively. DRG: dorsal root ganglia, GON: greater occipital nerve.

was significantly higher for the symptomatic side, at 0.649 \pm 0.204 , than that of the asymptomatic side, at $0.470 \pm 0.195$ $(P=0.003)$. In the C2-C3 DRG, the mean FA value in the symptomatic side was significantly lower than that in the asymptomatic side (C2: $0.176 \pm 0.059$ vs. $0.267 \pm 0.049, P$ $<0.001$; C3: $0.182 \pm 0.051$ vs. $0.261 \pm 0.048, P<0.001)$. The mean ADC value in the symptomatic side was significantly higher than that in the asymptomatic side (C2: $1.095 \pm$ 0.220 vs. $0.772 \pm 0.300, P<0.001$; C3: $1.038 \pm 0.295$ vs. 0.775 $\pm 0.294, P=0.003$ ) (Table 2).

\section{Intra/inter-observer performances}

There was excellent agreement between the inter- and intra-observer measurements of the FA in the GON and DRG (ICC $=0.861-0.964)$. Similarly, the agreement between the inter- and intra-observer measurements of the ADC in the GON and DRG (ICC $=0.909-0.967$ ) were excellent (Table 2).

\section{The results of the ROC curve analysis for the headache severity}

The ROC curves of FA and ADC are shown in Fig. 6A, 6B, respectively. The grading results of the DTI parameters for headache severity are summarized in Table 3 . Due to the highest AUC (0.866 [95\% CI: 0.767-0.965]) and highest Yoden index (0.743), the FA value of the C2 DRG was considered as the grading parameter for headache severity. The cut-off value was 0.205 with a sensitivity of 0.743 , specificity of 0.999, PPV of 0.933 and NPV of 0.857 .

\section{Correlation of the $\mathrm{HI}$ and the course of the disease with the $\mathrm{FA}$, and $\mathrm{ADC}$ values}

Bilateral FA and ADC values correlated with the HI. There was a significant negative correlation between the FA value of the GON and the $\mathrm{HI}(\mathrm{r}=-0.420, P=0.037)$. Instead, there was a significant positive correlation between the ADC values of the GON and the HI ( $r=0.531, P=0.006)$ (Table 4, Fig. 7). There was no significant correlation be- 
Table 3. The Grading Results for Headache Severity Using DTI Parameters

\begin{tabular}{|c|c|c|c|c|c|}
\hline Variable & AUC (95\% Cl) & Sensitivity & Specificity & Cut-off value & Yoden index ${ }^{\mathrm{a}}$ \\
\hline \multicolumn{6}{|l|}{ FA } \\
\hline C2 DRG & $0.866 *(0.767-0.965)$ & 0.743 & 0.999 & 0.205 & 0.743 \\
\hline C3 DRG & $0.759 *(0.617-0.901)$ & 0.743 & 0.800 & 0.191 & 0.543 \\
\hline GON & $0.733 *(0.618-0.928)$ & 0.743 & 0.867 & 0.254 & 0.610 \\
\hline \multicolumn{6}{|l|}{$\mathrm{ADC}$} \\
\hline C2 DRG & $0.800 *(0.678-0.927)$ & 0.933 & 0.657 & 0.909 & 0.590 \\
\hline C3 DRG & $0.731 *(0.589-0.874)$ & 0.867 & 0.600 & 0.822 & 0.467 \\
\hline GON & $0.680 *(0.517-0.843)$ & 0.733 & 0.686 & 0.579 & 0.419 \\
\hline
\end{tabular}

DTI: diffusion tensor imaging, AUC: area under the curve, Cl: confidence interval, FA: fractional anisotropy, DRG: dorsal root ganglia, GON: greater occipital nerve, ADC: apparent diffusion coefficient.

${ }^{a}$ Sensitivity + specificity -1

$\star P<0.05$.

Table 4. Correlation between FA, ADC Value and HI, Course ${ }^{\text {a }}$

\begin{tabular}{lrcc}
\hline $\begin{array}{c}\text { Pearson correlation } \\
\text { coefficient }\end{array}$ & C2 DRG & C3 DRG & GON \\
\hline FA value & & & \\
vs. course & -0.019 & -0.040 & -0.096 \\
vs. HI & 0.015 & -0.263 & $-0.420^{*}$ \\
ADC value & & & \\
vs. course & 0.232 & -0.299 & -0.164 \\
vs. HI & -0.075 & 0.136 & $0.531^{*}$ \\
\hline
\end{tabular}

FA: fractional anisotropy, ADC: apparent diffusion coefficient, HI: headache index, DRG: dorsal root ganglia, GON: greater occipital nerve.

${ }^{a}$ The value $=$ symptomatic value - asymptomatic value. $\star P<0.05$.

tween DTI parameters and course of disease.

\section{DISCUSSION}

According to the criteria of the IHS proposed in 2004 [20], CGH diagnosis requires radiological evidence. We obtained GON tractography with reliability and performed a quantified analysis using DTI parameters from the C2-C3 DRG and GON, which were considered vulnerable to neuropathy in CGH $[21,22]$. We found that the DTI parameter of the FA value for the C2 DRG can be used for detecting severe headaches with high accuracy. And we investigated the correlation between the DTI parameters of the nerves and clinical characteristics.

First, compared to the DRG, the GON was too small to be directly detected or identified on normal axial $\mathrm{T}_{1} \mathrm{WI}$ or $\mathrm{T}_{2} \mathrm{WI}$, let alone to set the ROI. Instead of quantitatively analyzing or observing the signal changes, we used $\mathrm{T}_{2} \mathrm{WI}$ CUBE reconstruction to locate the GON on superimposed morphological axial $\mathrm{T}_{1} \mathrm{WI}$ in order to accurately set the ROI. Imaging such an anatomical region with diffusionweighted imaging is not a trivial matter, given that the proximal GON is frequently hindered by susceptibility artifacts in the foramina. When setting the ROI on this segment, we selected a slice far away from the foramina and close to the lower edge of the inferior oblique muscle. A voxel-based analysis of the DTI data may be affected by possible partial volume effects (nerve size vs. voxel size). However, the ROI was a few square millimeters with a pixel value of 4-9 and thickness of $1.8 \mathrm{~mm}$, and was delineated according to the cross-sectional shape of the GON on axial $\mathrm{T}_{1} \mathrm{WI}$. The ROIs, in voxels, with respect to the DTI acquisition, were small due to the small size of the ROI on the GON. Kastler et al. [13] set the ROI in the direct visualization of the GONs on superimposed morphological axial $\mathrm{T}_{2} \mathrm{WI}$, and then depicted the GON tractography in high quality using the "whole-neck" flowing method. The direction of the 32 tracts prevented the uniformity of the diffusion-weighted gradient, and ensured an acceptable signal-to-noise ratio (SNR) with an examination time of about 15 minutes. For the present study, we acquired 6 DTI directions with a NEX of 6 for an acceptable SNR, with a shorter examination time of about 6 min. The study by Kastler et al. [13] showed good inter-observer agreement. In our study, the excellent ICC values of the intra-observer and inter-observer agreements showed the repeatability of DTI parameters at sections of the GONs on both sides and the C2-C3 DRG. Although setting the ROI can be a challenge in clinical practice, and also user-dependent without precise anatomical knowledge of the GON and DRG, it is vital for the reliability of DTI measurements.

Second, we found that the mean FA values in symptomatic side of GON and DRG were significantly lower than those in the respective asymptomatic side. Instead, the mean ADC values in the symptomatic side of the GON and DRG were significantly higher than those in the respective asymptomatic side. The isotropic situation in Wallerian degeneration, intrafascicular edema, and endo-/epi-neural swelling expanded the space between axons and nerve fascicles, as well as the extracellular matrix. Intraneural edema, axon swelling, myelinolysis and dilated intercel- 

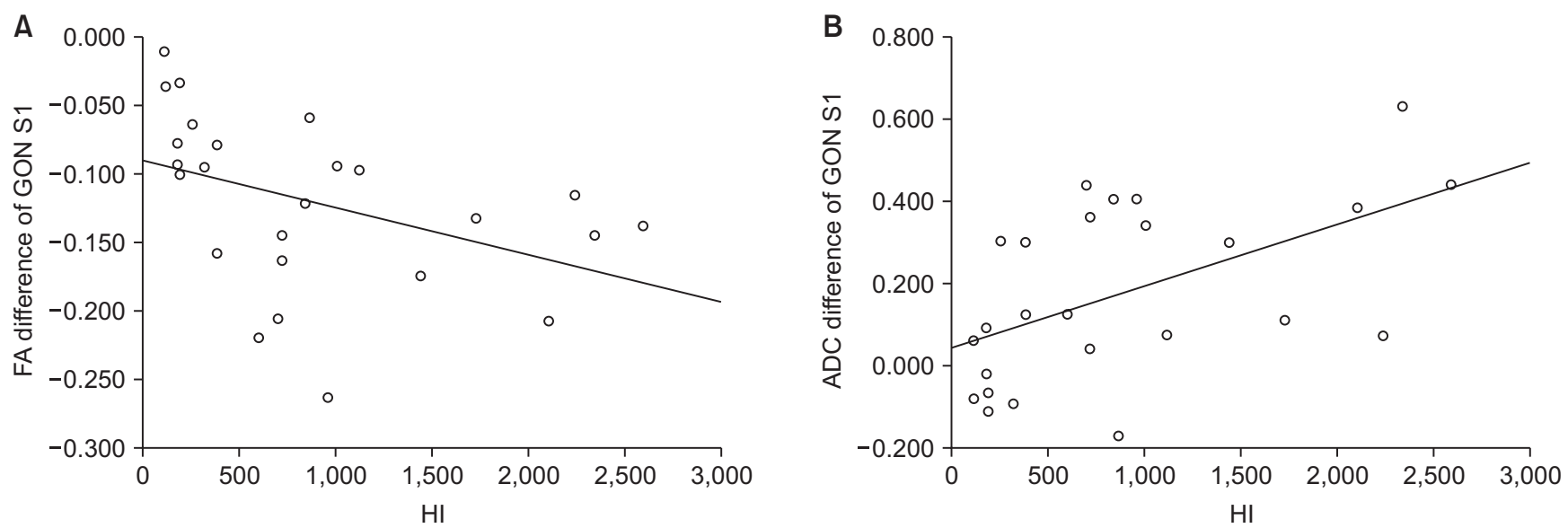

Fig. 7. Graphs showed the correlation between diffusion tensor imaging parameters of the nerves and $\mathrm{HI}$. The fractional anisotropy (FA) value in proximal greater occipital nerve (GON) was negatively correlated with headache index $(\mathrm{HI})(\mathrm{A}), r=-0.420, P=0.037$. The apparent diffusion coefficient $(\mathrm{ADC})$ value was positively correlated with $\mathrm{HI}(\mathrm{B}), \mathrm{r}=0.531, P=0.006$.

lular space, and increased diffusion of water molecules result in a high ADC value $[12,23,24]$. The results revealed neuropathy in the DRG in CGH, which were similar with results by Bovaira et al. [7] and Mehnert and Freedman [25]. We hypothesize that joint degeneration, connective tissue thickening, and fibrosis around nerves that affect the upper three DRG (not only near the C2 spinal nerve or C2 DRG) could be causes of CGH. Also, although imaging studies evaluating the function of the DRG using DTI are rare, we concluded that the DTI parameters changed due to the variable situation of neuropathy in the DRG. The GON block is one of the most common therapies for CGH, but its long-term efficacy is not good. The target of GON infiltration or a GON block using the landmark technique was very near the larger medial branch close to the obliquus capitis inferior muscle [26]. This point may be distant from the culprit proximal segment in patients with CGH [4]. As for neuropathy in the DRG in unilateral CGH, pulsed radiofrequency treatment of the DRG (especially C2 DRG), with or without a GON block, is widely used [6-8]. However, further studies are needed to resolve important remaining questions, such as, 'should we pay attention to infiltration of the proximal GON segments or the DRG segments?' and 'what are the neurography changes after interventional treatment of GON and DRG in CGH patients?'.

Third, we found a novel way to confirm headache severity using DTI for CGH. With high sensitivity, specificity, PPV, and NPV, the FA value in the C2 DRG for grading headache severity showed that neuropathy of this DRG was potentially correlated with the headache intensity in CGH, although the mechanisms are unclear. Headache with severe intensity lead to a higher analgesic consumption and a lower response rate for GON block treatment $[16,27]$. For patients who need invasive treatment, DTI for the target DRG is a promising method to evaluate the neu- ropathy as well as previous treatment planning. Especially for the patients undergoing long-time analgesic treatment, DTI parameters can reflect and predict the neuropathy of the DRG in CGH according to headache severity objectively. For ADC values, we also found an AUC of 0.800 in the C2 DRG, which was lower than that of the FA value in the C2 DRG. So we only concluded that the FA value in the C2 DRG was the parameter for grading headache severity. This was similar with the result by Wang et al. [12], who found that FA value is more reliable than ADC to diagnose nerve entrapment and is correlated with severity.

Finally, we found that with the increase in the HI, the FA decreases while the ADC increases in the proximal GON. Hwang et al. [28] found no correlation between the diameter, SNR, contrast-to-noise ratio and the course of the disease in the symptomatic side, but found significant negative correlation between the $\mathrm{T}_{2}$ signal intensity and course of the disease. Chen et al. [24] reported that the International Standards for Neurological Classification of Spinal Cord Injury score did not correlate with the FA value, but correlated positively with mean diffusivity, axial diffusivity, and radial diffusivity values in the nerves roots in the symptomatic side in patients with cervical disc herniation. However, they did not assess the correlation of FA and ADC difference with the clinical score. The potential microstructural changes from compressing nerve roots may be concealed. Due to the complex overlapping and distribution of cervical nerve branches in CGH [29], we concluded that when evaluating the correlation between DTI parameters and neuropathy, the FA and ADC difference in the bilateral nerves is required.

This study has certain limitations. First, we have not performed an imaging quality check of every patient, although we believe imaging was high quality. Second, neurography using DTI of other related occipital nerves 
should pay attention to this point, particularly for a nerve whose section is likely smaller than the voxel size. Third, the partial volume effects may affect the parameters of the small structures. A DTI sequence with a thinner slice and higher SNR is required for occipital nerves. Finally, to evaluate subtle contra-lateral GON injuries, a larger population with a true control group is needed.

In conclusion, for patients with unilateral CGH, DTI parameters of the GON and DRG were reliable. DTI of the symptomatic C2-C3 DRG and proximal GON revealed decreased FA value and increased ADC values. The FA value in the C2 DRG can be the grading parameter for headache severity. The FA value in the proximal GON was negatively correlated with $\mathrm{HI}$, whereas ADC value was positively correlated with $\mathrm{HI}$.

\section{ACKNOWLEDGMENTS}

The authors would like to thank Dr. Jixin Liu and Dr. Yofre C. for language editing.

\section{CONFLICT OF INTEREST}

No potential conflict of interest relevant to this article was reported.

\section{FUNDING}

No funding to declare.

\section{ORCID}

Lang Wang, https://orcid.org/0000-0002-0115-5173

Jiang Shen, https://orcid.org/0000-0003-0253-4252

Sushant Das, https://orcid.org/0000-0001-5128-4953

Hanfeng Yang, https://orcid.org/0000-0003-4527-127X

\section{REFERENCES}

1. Yao X, Lin X. [Warming-needle moxibustion for cervical headache: a randomized controlled trial]. Zhongguo Zhen Jiu 2016; 36: 463-6. Chinese.

2. Wang E, Wang D. Treatment of cervicogenic headache with cervical epidural steroid injection. Curr Pain Headache Rep 2014; 18: 442.

3. Pingree MJ, Sole JS, O'Brien TG, Eldrige JS, Moeschler SM. Clinical efficacy of an ultrasound-guided greater occipital nerve block at the level of C2. Reg Anesth Pain Med 2017; 42: 99-104.

4. Zipfel J, Kastler A, Tatu L, Behr J, Kechidi R, Kastler B. Ultrasound-guided intermediate site greater occipital nerve infiltration: a technical feasibility study. Pain Physician 2016; 19: E1027-34.

5. Sapunar D, Kostic S, Banozic A, Puljak L. Dorsal root ganglion - a potential new therapeutic target for neuropathic pain. J Pain Res 2012; 5: 31-8.

6. Lee SY, Jang DI, Noh C, Ko YK. Successful treatment of occipital radiating headache using pulsed radiofrequency therapy. J Korean Neurosurg Soc 2015; 58: 89-92.

7. Bovaira M, Peñarrocha M, Peñarrocha $M$, Calvo A, Jiménez A, March R. Radiofrequency treatment of cervicogenic headache. Med Oral Patol Oral Cir Bucal 2013; 18: e293-7.

8. Gabrhelík T, Michálek P, Adamus M. Pulsed radiofrequency therapy versus greater occipital nerve block in the management of refractory cervicogenic headache - a pilot study. Prague Med Rep 2011; 112: 279-87.

9. Sjaastad O, Fredriksen TA, Pfaffenrath V. Cervicogenic headache: diagnostic criteria. The Cervicogenic Headache International Study Group. Headache 1998; 38: 442-5.

10. Brienza M, Pujia F, Colaiacomo MC, Anastasio MG, Pierelli F, Di Biasi C, et al. 3T diffusion tensor imaging and electroneurography of peripheral nerve: a morphofunctional analysis in carpal tunnel syndrome. J Neuroradiol 2014; 41: 124-30.

11. Naraghi A, da Gama Lobo L, Menezes R, Khanna M, Sussman M, Anastakis D, et al. Diffusion tensor imaging of the median nerve before and after carpal tunnel release in patients with carpal tunnel syndrome: feasibility study. Skeletal Radiol 2013; 42: 1403-12.

12. Wang H, Ma J, Zhao L, Wang Y, Jia X. Utility of MRI diffusion tensor imaging in carpal tunnel syndrome: a meta-analysis. Med Sci Monit 2016; 22: 736-42.

13. Kastler A, Attye A, Heck O, Tahon F, Boubagra K, Tropes I, et al. Greater occipital nerve MR tractography: feasibility and anatomical considerations. J Neuroradiol 2018; 45: 54-8.

14. Wang L, Das S, Yang H. DTI of great occipital nerve neuropathy: an initial study in patients with cervicogenic headache. Clin Radiol 2019; 74: 899.el-899.e6.

15. Sjaastad O, Saunte C, Hovdahl H, Breivik H, Grønbaek E. "Cervicogenic" headache. An hypothesis. Cephalalgia 1983; 3: $249-56$.

16. Dilli E, Halker R, Vargas B, Hentz J, Radam T, Rogers R, et al. Occipital nerve block for the short-term preventive treatment of migraine: a randomized, double-blinded, placebocontrolled study. Cephalalgia 2015; 35: 959-68.

17. Määttä JH, Karppinen J, Paananen M, Bow C, Luk KD, Cheung KM, et al. Refined phenotyping of Modic changes: imaging biomarkers of prolonged severe low back pain and disability. Medicine (Baltimore) 2016; 95: e3495.

18. Cesmebasi A, Muhleman MA, Hulsberg P, Gielecki J, Matusz 
P, Tubbs RS, et al. Occipital neuralgia: anatomic considerations. Clin Anat 2015; 28: 101-8.

19. Das SK, Yang DJ, Wang JL, Zhang C, Yang HF. Non-Gaussian diffusion imaging for malignant and benign pulmonary nodule differentiation: a preliminary study. Acta Radiol 2017; 58: 19-26.

20. Headache Classification Subcommittee of the International Headache Society. The International Classification of Headache Disorders: 2nd edition. Cephalalgia 2004; 24 Suppl 1: 9-160.

21. Chua NH, Vissers KC, Sluijter ME. Pulsed radiofrequency treatment in interventional pain management: mechanisms and potential indications-a review. Acta Neurochir (Wien) 2011; 153: 763-71.

22. Denton TR, Shields LBE, Howe JN, Shanks TS, Spalding AC. Practical considerations of linear accelerator-based frameless extracranial radiosurgery for treatment of occipital neuralgia for nonsurgical candidates. J Appl Clin Med Phys 2017; 18: 123-32.

23. Zhang J, Zhang F, Xiao F, Xiong Z, Liu D, Hua T, et al. Quantitative evaluation of the compressed L5 and S1 nerve roots in unilateral lumbar disc herniation by using diffusion tensor imaging. Clin Neuroradiol 2018; 28: 529-37.

24. Chen YY, Lin XF, Zhang F, Zhang X, Hu HJ, Wang DY, et al.
Diffusion tensor imaging of symptomatic nerve roots in patients with cervical disc herniation. Acad Radiol 2014; 21: 338-44.

25. Mehnert MJ, Freedman MK. Update on the role of z-joint injection and radiofrequency neurotomy for cervicogenic headache. PM R 2013; 5: 221-7.

26. Nair AS, Kodisharapu PK, Anne P, Saifuddin MS, Asiel C, Rayani BK. Efficacy of bilateral greater occipital nerve block in postdural puncture headache: a narrative review. Korean J Pain 2018; 31: 80-6.

27. Gandolfi M, Donisi V, Marchioretto F, Battista S, Smania N, Del Piccolo L. A prospective observational cohort study on pharmacological habitus, headache-related disability and psychological profile in patients with chronic migraine undergoing onabotulinumtoxinA prophylactic treatment. Toxins (Basel) 2019; 11: 504.

28. Hwang L, Dessouky R, Xi Y, Amirlak B, Chhabra A. MR neurography of greater occipital nerve neuropathy: initial experience in patients with migraine. AJNR Am J Neuroradiol 2017; 38: 2203-9.

29. Bogduk N, Govind J. Cervicogenic headache: an assessment of the evidence on clinical diagnosis, invasive tests, and treatment. Lancet Neurol 2009; 8: 959-68. 